\title{
MODELAGEM CINÉTICA DA HIDRÓLISE ENZIMÁTICA DA PALHA DE CANA-DE-AÇÚCAR PRÉ-TRATADA
}

\author{
J. D. ANGARITA ${ }^{1}$, R. B. A. SOUZA², A. J. G. CRUZ ${ }^{2}$, E. C. BISCAIA Jr ${ }^{1}$ e A. R. SECCHI ${ }^{1}$ \\ ${ }^{1}$ Universidade Federal de Rio de Janeiro, COPPE, Programa de Engenharia Química \\ ${ }^{2}$ Universidade Federal de São Carlos, Departamento de Engenharia Química \\ E-mail para contato: arge@peq.coppe.ufrj.br
}

\begin{abstract}
RESUMO - Um modelo semimecanístico multireacional foi usado para descrever a hidrólise enzimática da palha de cana de açúcar (PCA) pré-tratada hidrotermicamente. O modelo considera a reação de conversão homogênea da celobiose em glicose, duas reações heterogêneas para a conversão de celulose em celobiose e glicose, conversão da hemicelulose em xilose, inibição competitiva da enzima pelos produtos da hidrólise e uma isoterma tipo Langmuir para descrever a adsorção da enzima no substrato. O software EMSO foi utilizado para estimar os parâmetros do modelo a partir de uma condição de referência (15\% m/v de sólidos e 10 FPU/g-celulose de enzima). O modelo mostrou ter capacidade para predizer o comportamento da hidrólise de PCA em diferentes condições de carga de sólidos (10-20\% m/v), concentrações de enzima (5-60FPU/g-celulose) e cargas iniciais de açúcares (glicose de 30 e 60g/l, celobiose e xilose de 10g/l). Uma simplificação nos parâmetros do modelo, baseada nos fundamentos da cinética enzimática mostrou ser uma boa opção para superar a alta correlação paramétrica referida na literatura para este tipo de modelo, sem afetar a acurácia nas predições. O modelo tem o potencial de ser utilizado em aplicações de projeto de reatores, simulação e otimização dentro do processo de produção do etanol de segunda geração.
\end{abstract}

\section{INTRODUÇÃO}

A modelagem matemática da hidrólise enzimática de materiais lignocelulósicos é um tema desafiante na engenharia, refletido no grande número de modelos propostos (Bansal et al., 2009). Na hidrólise enzimática ocorrem reações de múltiplos estágios em sistema heterogêneo onde a celulose insolúvel é inicialmente degradada na interface sólido-líquido pela ação sinérgica do pool de enzimas endoglicanases (EG) e exo-glicanases/celobiohidrolases ( $\mathrm{CBH}^{\prime}$ s), acompanhada da hidrólise na fase líquida dos produtos intermediários solúveis (celobiose e cadeias curtas de oligossacarídeos) que são convertidos em glicose pelas $\beta$-glicosidases (BG) (Zhang e Lynd, 2004).

Em geral, a hidrólise depende das características das enzimas como: (1) adsorção da enzima no substrato, (2) inibição competitiva ou não competitiva pelo produto final, (3) sinergismo e (4) limitações da transferência de massa que afetam o transporte da enzima para o substrato. A hidrólise é também afetada pelas características do substrato como: (1) composição e distribuição dos componentes do substrato (lignina, hemicelulose, proteínas e gorduras), (2) tamanho de partícula e (3) 


\section{9 a 22 de outubro de 2014 \\ Florianópolis/SC}

a cristalinidade (Brown et al., 2010). Diferentes modelos semimecanísticos têm sido propostos para descrever a hidrólise enzimática de biomassa pré-tratada (Bansal et al., 2009; Zhang e Lynd, 2004). Kadam et al. (2004) desenvolveram e validaram um modelo cinético para a hidrólise enzimática em batelada de palha de milho pré-tratada com ácido diluído. Este modelo considerou uma reação homogênea de celobiose a glicose e duas reações heterogêneas de celulose a celobiose e a glicose. A adsorção da enzima foi incorporada usando uma isoterma tipo Langmuir; a inibição competitiva pelos produtos finais da hidrólise e a reatividade do substrato foram incluídas no modelo. Uma extensão deste modelo foi proposta por Câmara (2012) para levar em consideração tanto a formação de xilose a partir da hemicelulose, quanto a inibição da xilose sobre as enzimas de acordo com a Figura 1.

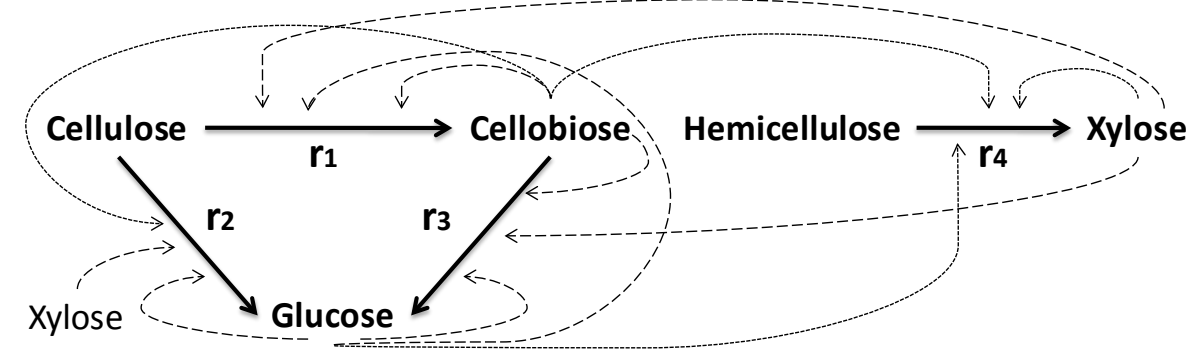

Figura 1 - Mecanismo cinético para a modelagem da hidrólise da celulose. Celulases (EG e CBH's) envolvidas em $\mathbf{r}_{\mathbf{1}}$ e $\mathbf{r}_{2}, \beta$-glicosidase em $\mathbf{r}_{\mathbf{3}}$ e hemicelulases em $\mathbf{r}_{\mathbf{4}}$. Tanto as linhas pontilhadas quanto $\mathbf{r}_{4}$ foram propostas por Câmara (2012), modificado de Kadam et al. (2004).

Nos últimos dez anos, o modelo de Kadam et al. (2004) tem sido verificado experimentalmente (Zheng et al., 2009; Hodge et al., 2009), analisado estatisticamente (Sin et al., 2010), avaliado em aplicações de otimização, controle e configurações de processo (Hodge et al., 2009; MoralesRodríguez et al., 2010, 2011; Mutturi e Lidén, 2013), e usado recentemente na modelagem da hidrólise enzimática do bagaço de cana-de-açúcar (Câmara, 2012).

Embora a hidrólise enzimática tenha vantagens sobre a conversão química de alto rendimento de açúcar como o baixo consumo de energia, condições de operação brandas (pressão e temperaturas baixas), biorreatores de baixo custo e formação mínima de subprodutos (Sun e Cheng, 2005), o custo da hidrólise enzimática da biomassa lignocelulósica é uma das principais barreiras da comercialização do etanol de segunda geração (Zheng, 2009). Uma das abordagens para reduzir esses custos é a operação em alta concentração de sólidos, geralmente apresentada com cargas de sólidos $>15 \%$ (Hodge et al., 2009). A hidrólise enzimática em alta concentração de sólidos apresenta vantagens econômicas em relação ao processo convencional em baixa concentração de sólidos, como: (1) menor custo de capital devido à redução do volume; (2) menor custo de operação devido à menor quantidade de energia para aquecer e resfriar; (3) menor custo na etapa de separação do produto devido às altas concentrações de produto, e (4) redução dos custos de eliminação e tratamento de resíduos devido ao baixo consumo de água (Mohagheghi et al., 1992), porém, existem poucos modelos e dados experimentais da hidrólise de biomassa lignocelulósica em alta concentração de sólidos.

Este trabalho teve como objetivo desenvolver a partir de dados experimentais, um modelo suficientemente sofisticado para descrever a hidrólise enzimática da PCA pré-tratada em alta 


\section{9 a 22 de outubro de 2014 \\ Florianópolis/SC}

concentração de sólidos, a partir das propriedades macroscópicas do sistema, com o potencial de ser utilizado tanto no projeto de reatores, quanto em aplicações de simulação, otimização e controle no processo de produção do etanol lignocelulósico.

\section{MATERIAIS E MÉTODOS}

Os dados experimentais para a estimação dos parâmetros cinéticos e a verificação do modelo, foram gerados a partir de ensaios de hidrólise enzimática com PCA pré-tratada hidrotermicamente a $195^{\circ} \mathrm{C}$ por $10 \mathrm{~min}$, seguindo as melhores condições de pré-tratamento descritas por Souza e Cruz (2013). O complexo enzimático comercial Cellic ${ }^{\circledR}$ CTec2 (203 FPU/ml, 36 mg-proteína/ml; Novozymes, Araucária, PR) foi usado nos experimentos sem adição de $\beta$-glicosidase. Outras condições são listadas na Tabela 1.

Tabela 1 - Condições experimentais para o desenvolvimento e validação do modelo cinético ${ }^{\mathrm{a}}$.

\begin{tabular}{|c|c|c|c|c|c|c|}
\hline \multirow[b]{3}{*}{ Condições Experimentais } & \multicolumn{6}{|c|}{ Experimentos } \\
\hline & \multirow{2}{*}{$\begin{array}{l}\text { Condição de } \\
\text { Referência }\end{array}$} & \multicolumn{5}{|c|}{ Condições de validação } \\
\hline & & $\begin{array}{c}\text { Carga de } \\
\text { Enzima } \\
\end{array}$ & $\begin{array}{l}\text { Carga de } \\
\text { Sólidos } \\
\end{array}$ & $\begin{array}{l}\text { Glicose } \\
\text { inicial }\end{array}$ & $\begin{array}{c}\text { Celobiose } \\
\text { inicial }\end{array}$ & $\begin{array}{l}\text { Xilose } \\
\text { Inicial }\end{array}$ \\
\hline $\begin{array}{l}\text { Carga de enzima } \\
\text { (FPU/g-celulose) }\end{array}$ & 10 & $\begin{array}{c}5,15,20,25 \\
30,60\end{array}$ & 10 & 10 & 10 & 10 \\
\hline Carga de sólidos (\%m/v) & 15 & 15 & 10,20 & 15 & 15 & 15 \\
\hline Glicose inicial (g/l) & 0 & 0 & 0 & 30,60 & 0 & 0 \\
\hline Celobiose inicial (g/l) & 0 & 0 & 0 & 0 & 10 & 0 \\
\hline Xilose inicial (g/l) & 0 & 0 & 0 & 0 & 0 & 10 \\
\hline
\end{tabular}

Amostras do sobrenadante foram coletadas em tempos de amostragem de 1, 2, 6, 12, 24 , 48 e 72h (amostras adicionais foram coletadas para a condição de referência em $t=4$, 8 e 96h) para medições de concentração de glicose, celobiose e xilose pela técnica de HPLC. A concentração de proteína em solução foi medida pelo ensaio de proteínas de Bradford. Experimentos para a verificação do modelo foram conduzidos para diferentes cargas de enzima (5-60 FPU/g-celulose), cargas de sólidos (10 e 20\% m/v) e diferentes concentrações iniciais de açúcares, incluindo glicose (30 e 60 g/l), celobiose $(10 \mathrm{~g} / \mathrm{l})$ e xilose $(10 \mathrm{~g} / \mathrm{l})$. Os dados experimentais em condições diferentes das condições de referência foram utilizados para validar as previsões do modelo e para avaliar a fidelidade do modelo ao descrever o efeito inibidor dos açúcares. Todos os experimentos foram realizados no Laboratório de Desenvolvimento e Automação de Bioprocessos da Universidade Federal de São Carlos.

\subsection{Desenvolvimento do Modelo}

A Figura 1 apresenta um esquema da hidrólise da celulose em glicose baseado em três reações $\left(r_{1}, r_{2}, r_{3}\right)$. Uma reação a mais $\left(r_{4}\right)$ foi considerada para modelar a hidrólise da hemicelulose em xilose, seguindo a proposta de Câmara (2012). As equações do modelo são listadas na Tabela 2.

O complexo enzimático foi modelado como uma pseudo-enzima com diferentes atividades 
enzimáticas, sem distinção entre celulose amorfa e cristalina. A atividade enzimática foi considerada proporcional à concentração de proteína na fase aquosa (Ooshima et al., 1990), considerou-se que a composição na superfície varia pouco durante a hidrólise (Liao et al., 2008), a adsorção foi descrita por isoterma tipo Langmuir, a conversão de celobiose em glicose em solução foi descrita pela cinética de Michaelis-Menten e a inativação térmica e mecânica da enzima foi desprezada.

Tabela 2 - Modelo cinético da hidrólise enzimática de biomassa lignocelulósica

\begin{tabular}{|c|c|c|c|c|c|}
\hline \multicolumn{3}{|c|}{ Adsorção da enzima e equações de taxa } & \multicolumn{3}{|c|}{ Balanços de massa } \\
\hline $\begin{array}{l}\text { Isoterma de } \\
\text { adsorção tipo } \\
\text { Langmuir. }\end{array}$ & $\frac{E_{B}}{S}=\frac{E_{\max } K_{a d} E_{F}}{1+K_{a d} E_{F}}$ & (1) & Enzima total & $E_{T}=E_{F}+E_{B}$ & (8) \\
\hline $\begin{array}{l}\text { Enzima } \\
\text { adsorvida na } \\
\text { celulose (C). }\end{array}$ & $E_{B C}=E_{B} \frac{C}{S}$ & (2) & $\begin{array}{l}\text { Sólidos } \\
\text { insolúveis (S) }\end{array}$ & $S=C+H+L$ & (9) \\
\hline $\begin{array}{l}\text { Enzima } \\
\text { adsorvida na } \\
\text { hemicelulose } \\
\text { (H). }\end{array}$ & $E_{B H}=E_{B} \frac{H}{S}$ & (3) & Celulose & $\frac{d C}{d t}=-r_{1}-r_{2}$ & (10) \\
\hline $\begin{array}{l}\text { Conversão } \\
\text { da celulose } \\
\text { (C) em } \\
\text { celobiose } \\
\text { (G2). }\end{array}$ & $r_{1}=\frac{k_{1 r} E_{B C} R_{S} S}{1+\frac{G 2}{K_{1 I G 2}}+\frac{G}{K_{1 I G}}+\frac{X}{K_{1 I X}}}$ & (4) & Celobiose & $\frac{d G 2}{d t}=1,056 r_{1}-r_{3}$ & (11) \\
\hline $\begin{array}{l}\text { Conversão } \\
\text { da celulose } \\
\text { (C) em } \\
\text { glicose (G). }\end{array}$ & $r_{2}=\frac{k_{2 r} E_{B C} R_{S} S}{1+\frac{G 2}{K_{2 I G 2}}+\frac{G}{K_{2 I G}}+\frac{X}{K_{2 I X}}}$ & (5) & Glicose & $\frac{d G}{d t}=1,111 r_{2}+1,053 r_{3}$ & (12) \\
\hline $\begin{array}{l}\text { Conversão } \\
\text { da celobiose } \\
\text { (G2) em } \\
\text { glicose (G). }\end{array}$ & $r_{3}=\frac{k_{3 r} E_{F} G 2}{K_{3 M}\left(1+\frac{G}{K_{3 I G}}+\frac{X}{K_{3 I X}}\right)+G 2}$ & (6) & Hemicelulose & $\frac{d H}{d t}=-r_{4}$ & (13) \\
\hline $\begin{array}{l}\text { Conversão } \\
\text { da } \\
\text { hemicelulose } \\
\text { (H) em } \\
\text { xilose (X). }\end{array}$ & $r_{4}=\frac{k_{4 r} E_{B H} R_{S} S}{1+\frac{G 2}{K_{4 I G 2}}+\frac{G}{K_{4 I G}}+\frac{X}{K_{4 I X}}}$ & (7) & Xilose & $\frac{d X}{d t}=1,136 r_{4}$ & (14) \\
\hline
\end{tabular}

\subsection{Metodologia Computacional}

O software EMSO (Soares e Secchi, 2003) foi utilizado para estimar tanto os parâmetros de adsorção quanto os parâmetros cinéticos do modelo. Os parâmetros da isoterma tipo Langmuir foram estimados independentemente a partir dos dados experimentais de proteína no sobrenadante das 
hidrólises com diferentes cargas de enzima de acordo com a Tabela 1. A constante de reatividade do substrato $(\alpha)$ foi considerada unitária segundo o valor usado por Câmara (2012) para bagaço.

\section{RESULTADOS E DISCUSSÃO}

Os parâmetros foram estimados pelo método dos Mínimos Quadrados Ponderados usando o algoritmo dos poliedros flexíveis. Os dois conjuntos de parâmetros estimados (adsorção e cinéticos) são listados na Tabela 3 (na coluna Modelo Original).

Tabela 3 - Parâmetros estimados do modelo

\begin{tabular}{lc}
\hline Parâmetro & Valor \\
\hline$K_{a d}(\mathrm{l} / \mathrm{g})$ & Parâmetros de adsorção \\
$E_{\max }(\mathrm{g} / \mathrm{kg})$ & 7,16 \\
& 8,32
\end{tabular}

Parâmetros cinéticos obtidos a partir dos dados de sacarificação

\begin{tabular}{lccc} 
& Modelo Original & Modelo Simplificado & Com todos os dados* \\
$k_{1 r}(\mathrm{l} / \mathrm{g} / \mathrm{h})$ & 0,028 & 0,104 & 0,015 \\
$k_{2 r}(\mathrm{l} / \mathrm{g} / \mathrm{h})$ & 4,78 & 2,76 & 0,548 \\
$k_{3 r}(1 / \mathrm{h})$ & 187,8 & 143,2 & 170,9 \\
$k_{4 r}(\mathrm{l} / \mathrm{g} / \mathrm{h})$ & 15,66 & 21,42 & 6,85 \\
$K_{1 I G 2}(\mathrm{l} / \mathrm{g})$ & 0,545 & 1,98 & 6,02 \\
$K_{1 I G}(\mathrm{l} / \mathrm{g})$ & 2,93 & 0,546 & 3,57 \\
$K_{1 I X}(\mathrm{l} / \mathrm{g})$ & 5,07 & 1,60 & 5,45 \\
$K_{2 I G 2}(\mathrm{l} / \mathrm{g})$ & 117,2 & & \\
$K_{2 I G}(\mathrm{l} / \mathrm{g})$ & 0,329 & & \\
$K_{2 I X}(\mathrm{l} / \mathrm{g})$ & 0,325 & & \\
$K_{3 M}(\mathrm{l} / \mathrm{g})$ & 25,50 & 45,49 & 45,6 \\
$K_{3 I G}(\mathrm{l} / \mathrm{g})$ & 0,216 & 0,409 & 0,032 \\
$K_{3 I X}(\mathrm{l} / \mathrm{g})$ & 59,07 & 73,10 & 79,42 \\
$K_{4 I G 2}(\mathrm{l} / \mathrm{g})$ & 20.68 & & \\
$K_{4 I G}(\mathrm{l} / \mathrm{g})$ & 0.728 & & \\
$K_{4 I X}(\mathrm{l} / \mathrm{g})$ & 196.4 & & \\
\hline
\end{tabular}

* Parâmetros estimados com o Modelo Simplificado usando todos os dados experimentais disponíveis.

O conjunto de parâmetros do Modelo Original forneceu um bom ajuste para os dados experimentais na condição de referência conforme mostra a Figura 2a, porém, não foi possível estimar a significância, o intervalo de confiança (IC), e a matriz de correlação dos parâmetros, devido à alta correlação entre eles que gerou mau condicionamento da matriz de informação de Fisher. Sin et al. (2010) analisaram estatisticamente este modelo e observaram que todos os parâmetros têm IC muito grande, isto significa que os parâmetros são estatisticamente não identificáveis e que o modelo está sobreparametrizado em relação às medições disponíveis.

Baseado nos fundamentos da cinética enzimática uma simplificação do modelo foi proposta para superar a correlação paramétrica. Dado que o complexo enzimático foi modelado como uma pseudo-enzima com múltiplas atividades, as constantes de inibição das reações cinéticas que acontecem na interface sólido-líquido $\left(r_{1}, r_{2}\right.$ e $\left.r_{4}\right) K_{i I G 2}, K_{i I G}$ e $K_{i I X}(i=1,2$ e 4$)$ nas Eq. (4), (5) e (7), 
foram substituídas por um só conjunto de constantes de inibição ( $K_{I G 2}, K_{I G}$ e $\left.K_{I X}\right)$ que representam o efeito global da inibição dos produtos da hidrólise sobre as atividades da pseudo-enzima. Os parâmetros estimados para o modelo simplificado e o ajuste do modelo nas condições de referência são apresentados na Tabela 3 (na coluna Modelo Simplificado) e na Figura 2b, respectivamente.

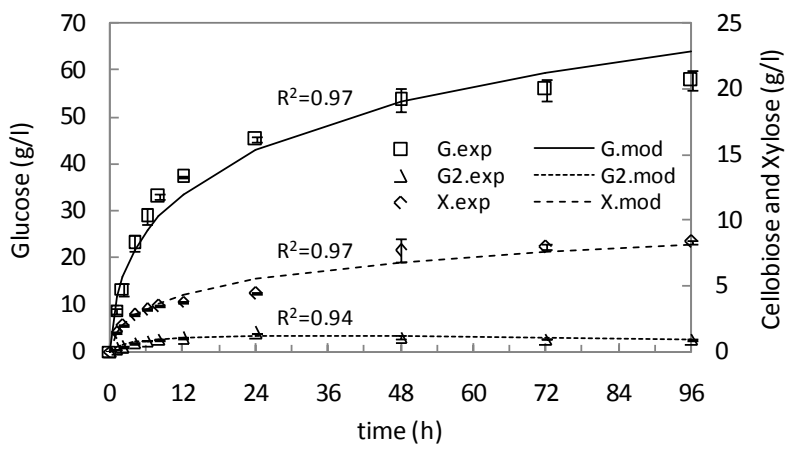

(a)

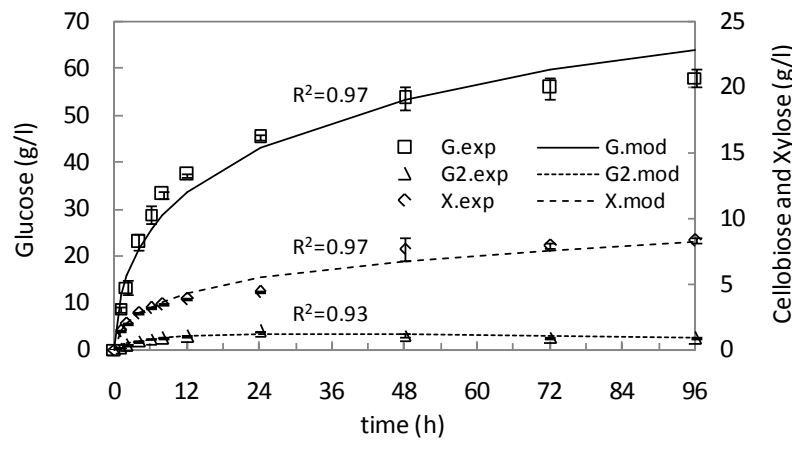

(b)

Figura 2 - Hidrólise enzimática de PCA pré-tratada nas condições de referência. (a) Modelo Original (com todos os parâmetros); (b) Modelo Simplificado.

De acordo com a Figura 2, o ajuste do modelo não foi afeitado pela simplificação no número de parâmetros. Embora o modelo simplificado permitisse avaliar a significância, IC e a matriz de correlação dos parâmetros (dados não apresentados), alguns parâmetros continuaram fortemente correlacionados (17 pares de parâmetros com coeficiente de correlação paramétrica $\left|\rho_{i j}\right|>0,90$ ). Assim, uma nova estimação de parâmetros foi feita na tentativa de reduzir a correlação usando todos os dados experimentais disponíveis. Os parâmetros obtidos e o ajuste do modelo na condição de referência são apresentados na Tabela 3 (na coluna "Com todos os dados") e na Figura 3, respectivamente.

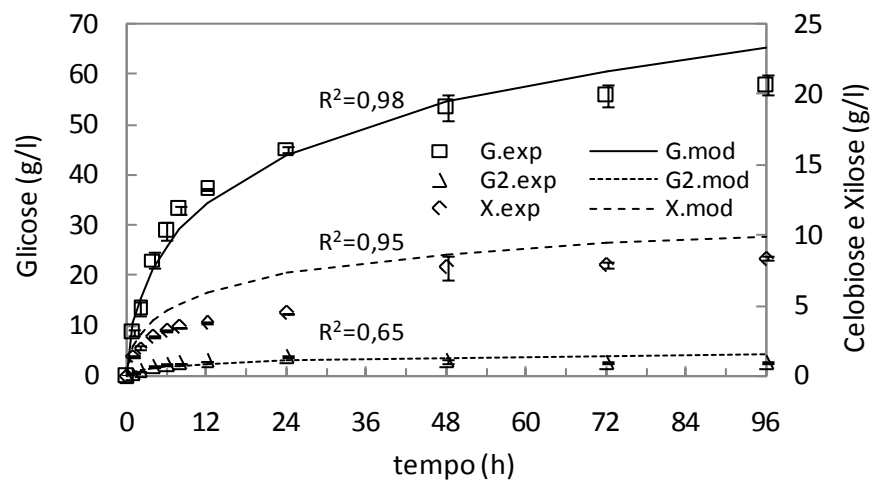

Figura 3 - Hidrólise enzimática de PCA pré-tratada nas condições de referência. Parâmetros estimados para o modelo simplificado com todos os dados experimentais disponíveis.

A nova estimação melhorou a significância e o IC dos parâmetros além de reduzir a correlação em 50\%. Apesar de ter degradado o ajuste para a concentração de celobiose na condição de referência, as predições de glicose e xilose continuaram sendo boas e foram melhoradas as predições de celobiose para outras condições experimentais como é apresentado na Tabela 4 (colunas sombreadas). 
Tabela 4 - Comparação do ajuste $\left(R^{2}\right)$ do modelo para diferentes condições experimentais

\begin{tabular}{|c|c|c|c|c|c|c|c|c|c|c|c|c|c|c|c|c|c|c|}
\hline \multicolumn{19}{|c|}{ Efeito da carga enzimática (FPU/g-celulose) } \\
\hline & \multicolumn{3}{|c|}{5} & \multicolumn{3}{|c|}{15} & \multicolumn{3}{|c|}{20} & \multicolumn{3}{|c|}{25} & \multicolumn{3}{|c|}{30} & \multicolumn{3}{|c|}{60} \\
\hline & G & G2 & $\mathbf{X}$ & G & G & $\mathbf{X}$ & G & G2 & $\mathbf{X}$ & G & G2 & $\mathbf{X}$ & G & G2 & $\mathbf{X}$ & G & G2 & $\mathbf{X}$ \\
\hline MO & 0,98 & 0,87 & 0,99 & 0,99 & 0,6 & 0,9 & 0,9 & 0,16 & 0,96 & 0,98 & 0,09 & 0,93 & 0,93 & 0,21 & 0,89 & 0,89 & 0,02 & 0,87 \\
\hline MS & 0,98 & 0,85 & 0,99 & 0,99 & 0,6 & 0,9 & 0,9 & 0,15 & 0,96 & 0,98 & 0,07 & 0,94 & 0,93 & 0,19 & 0,90 & 0,89 & 0,01 & 0,87 \\
\hline TE & 0,98 & 0,91 & 0,99 & 0,99 & 0,3 & 0,9 & 0,9 & 0,96 & 0,97 & 0,98 & 0,97 & 0,95 & 0,92 & 0,89 & 0,93 & 0,88 & 0,87 & 0,89 \\
\hline \multicolumn{7}{|c|}{ Efeito da carga de sólidos (\%m/v) } & \multicolumn{12}{|c|}{ Efeito da inibição pelos açúcares (g/l) } \\
\hline & \multicolumn{3}{|c|}{10} & \multicolumn{3}{|c|}{20} & \multicolumn{3}{|c|}{$10 X$} & \multicolumn{3}{|c|}{ 30G } & \multicolumn{3}{|c|}{ 60G } & \multicolumn{3}{|c|}{ 10G2 } \\
\hline & $\mathbf{G}$ & G2 & $\mathbf{X}$ & G & G2 & $\mathbf{X}$ & G & G2 & $\mathbf{X}$ & $\mathbf{G}$ & G2 & $\mathbf{X}$ & G & G2 & $\mathbf{X}$ & G & G2 & $\mathbf{X}$ \\
\hline MO & 0,96 & 0,38 & 0,83 & 0,94 & 0,67 & 0,99 & 0,97 & 0,54 & 0,82 & 0,87 & 0,82 & 0,74 & 0,91 & 0,08 & 0,43 & 0,96 & 0,83 & 0,75 \\
\hline MS & 0,96 & 0,35 & 0,83 & 0,94 & 0,66 & 0,99 & 0,96 & 0,56 & 0,85 & 0,88 & 0,83 & 0,75 & 0,91 & 0,06 & 0,44 & 0,96 & 0,78 & 0,75 \\
\hline TE & 0,96 & 0,78 & 0,86 & 0,94 & 0,82 & 0,97 & 0,96 & 0,85 & 0,87 & 0,87 & 0,87 & 0,80 & 0,91 & 0,04 & 0,50 & 0,96 & 0,77 & 0,79 \\
\hline
\end{tabular}

\section{CONCLUSÕES}

Um modelo semimecanístico multireacional simplificado foi gerado para descrever a hidrólise enzimática da palha de cana de açúcar pré-tratada hidrotermicamente. Além de melhorar a correlação paramétrica, o modelo simplificado mostrou ter capacidade de predizer o comportamento da hidrólise enzimática da PCA para diferentes condições de carga de sólidos, enzima e açúcares iniciais. Embora o modelo ainda tenha alguma dificuldade em estimar a celobiose e xilose sob o efeito inibitório da glicose $(60 \mathrm{~g} / \mathrm{l})$, o modelo prediz com boa acurácia a concentração de glicose $\left(R^{2}>0,87\right)$ e o importante efeito inibitório da enzima pelos produtos em altas concentrações de sólidos.

\section{AGRADECIMENTOS}

Os autores gostariam de agradecer a CAPES (Coordenação de Aperfeiçoamento de Pessoal de Nível Superior), ao CNPq (Conselho Nacional de Desenvolvimento Científico e Tecnológico) e à FAPERJ (Fundação de Amparo à Pesquisa do Estado do Rio de Janeiro) pelo suporte financeiro.

\section{REFERÊNCIAS}

BANSAL, P.; HALL, M.; REALFF, M. J.; LEE, J. H.; BOMMARIUS, A. S. Modeling cellulase kinetics on lignocellulosic substrates. Biotechnol. Adv., v. 27, p. 833-848, 2009.

BROWN, R. F.; AGBOGBO, F. K.; HOLTZAPPLE, M. T. Comparison of mechanistic models in the initial rate enzymatic hydrolysis of AFEX-treated wheat straw. Biotechnol. Biofuels, v.3:6, doi:10.1186/1754-6834-3-6, 2010.

CÂMARA, M. M. Modelagem e simulação da hidrólise de bagaço de cana pré-tratado com peróxido de hidrogênio em meio alcalino (Dissertação de M.Sc.). Universidade Estadual de Maringá, 2012. 


\section{9 a 22 de outubro de 2014 \\ Florianópolis/SC}

HODGE, D. B.; KARIM, M. N.; SCHELL, D. J.; McMILLAN, J. D. Model-based fed-batch for highsolids enzymatic cellulose hydrolysis. Appl. Biochem. Biotechnol., v.152, p. 88-107, 2009.

KADAM, K. L.; RYDHOLM, E. C.; McMILLAN, J. D. Development and validation of a kinetic model for enzymatic saccharification of lignocellulosic biomass. Biotechnol. Progr., v.20, p. 698-705, 2004.

LIAO, W.; LIU, Y.; WEN, Z.; FREAR, C.; CHEN, S. Kinetic modeling of enzymatic hydrolysis of cellulose in differently pretreated fibers from dairy manure. Biotechnol. Bioeng., v.101, p. 441-451, 2008.

MOHAGHEGHI, A.; TUCKER, M.; GROHMANN, K.; WYMAN, C. High solids simultaneous saccharification and fermentation of pretreated wheat straw to ethanol. Appl. Biochem. Biotechnol., v.33, p. 67-81, 1992.

MORALES-RODRIGUEZ, R.; CAPRON, M.; HUUSOM, J. K.; SIN, G. Controlled fed-batch operation for improving cellulose hydrolysis in 2G bioethanol production. 20th European Symposium on Computer Aided Process Engineering - ESCAPE20. Ischia, Naples, Italy, June 6-9, 2010.

MORALES-RODRIGUEZ, R.; MEYER, A. S.; GERNAEY, K. V.; SIN, G. Dynamic model-based evaluation of process configurations for integrated operation of hydrolysis and co-fermentation for bioethanol production from lignocellulose. Bioresour. Technol., v.102, p. 1174-1184, 2011.

MUTTURI, S.; LIDEN, G. Model-based estimation of optimal temperature profile during simultaneous saccharification and fermentation of Arundo donax. Biotechnol. Bioeng., doi:10.1002/bit.25165, 2013.

OOSHIMA, H., BURNS, D. S., CONVERSE, A. O. Adsorption of cellulase from Trichoderma reesei on cellulose and lignacious residue in wood pretreated by dilute sulfuric acid with explosive decompression. Biotechnol. Bioeng., v.36, p. 446-452, 1990.

SIN, G.; MEYER, A. S.; GERNAEY, K. V. Assessing reliability of cellulose hydrolysis models to support biofuel process design-Identifiability and uncertainty analysis. Comput. Chem. Eng., v.34, p. 1385-1392, 2010.

SOARES, R. P.; SECCHI, A. R. EMSO: A new environment for modelling, simulation and optimisation. Comput. Aided Chem. Eng., v.14, p. 947-952, 2003.

SOUZA, R. B. A.; CRUZ, A. J. G. Pré-tratamento, hidrólise enzimática e fermentação da palha de cana-de-açúcar. XIX simpósio nacional de bioprocessos e $X$ simpósio de hidrólise enzimática de biomassas, Foz de Iguaçu, Brasil, 30 de julho-02 de agosto, 2013.

SUN, Y.; CHENG, J. J. Dilute acid pretreatment of rye straw and bermuda grass for ethanol production. Bioresour. Technol., v.96, p. 1599-1606, 2005.

ZHANG, Y-H. P.; LYND, L. R. Toward an aggregated understanding of enzymatic hydrolysis of cellulose: Noncomplexed cellulase systems. Biotechnol. Bioeng., v. 88, p. 797-824, 2004.

ZHENG, Y.; PAN Z.; ZHANG, R.; JENKINS, B. M. Kinetic modeling for enzymatic hydrolysis of pretreated creeping wild ryegrass. Biotechnol. Bioeng., v.102, p. 1558-1569, 2009. 